\title{
Improving the External Quantum Efficiency of High Power GaN Based Flip-Chip LEDs using Ag/SiO2/DBR/SiO2 Composite ReflectiveStructure
}

Liang Xu

South China Normal University

Jinglin Zhan

Peking University

Huiqing Sun

South China Normal University

Zhizhong Chen

Peking University

Zhiyou Guo ( $\nabla$ guozy@scnu.edu.cn )

South China Normal University

\section{Research Article}

Keywords: flip-chip LEDs (FCLEDs), Ag/SiO2/distributed Bragg, composite reflection structure (CRS), nGaN

Posted Date: February 17th, 2021

DOI: https://doi.org/10.21203/rs.3.rs-204748/v1

License: (c) (1) This work is licensed under a Creative Commons Attribution 4.0 International License. Read Full License 


\title{
Improving the External Quantum Efficiency of High Power GaN Based Flip-Chip LEDs using $\mathrm{Ag} / \mathrm{SiO}_{2} / \mathrm{DBR} / \mathrm{SiO}_{2}$ Composite Reflective Structure
}

\author{
Liang $\mathrm{Xu}^{1}$, Jinglin Zhan ${ }^{2}$, Huiqing Sun', Zhizhong $\mathrm{Chen}^{2}$ and Zhiyou Guo'
}

Improve the light extraction efficiency and light output in the vertical direction of LEDs for highpower applications, flip-chip LEDs (FCLEDs) with an $\mathrm{Ag} / \mathrm{SiO}{ }_{2} /$ distributed $\mathrm{Bragg}$ reflector/SiO composite reflection structure (CRS) were fabricated. The enhanced opto-electrical properties were thoroughly investigated. Compared with the normal Ag-based FCLEDs, the light output power of the CRS-FCLEDs is increased by $6.3 \%$ at an operational current of $1500 \mathrm{~mA}$, with the corresponding external quantum efficiency improved by $6.0 \%$. Further investigation proved that the CRS structure exhibited higher reflectance compared with the commonly used Agmirror reflective structure, which originates from the increased reflective area in the sidewall and partial area of the $n-G a N$ contact holes. Moreover, the light emission intensity distributions and far-field angular light emission measurements show that the CRS-FCLED has a strengthened light output in the vertical direction, which shows great potential for applications in high-power fields, such as headlamps for automobiles.

With the development of semiconductor materials and packaging technology, the luminous flux of high-power white LEDs has rapidly improved. The application of LEDs in the automotive lighting field has gradually expanded, from signal indicators to current automotive headlights, reflecting the development trend that LEDs will become the mainstream light source in this field ${ }^{[1]-[4]}$.

Flip-chip LEDs (FCLEDs) exhibit excellent heat dissipation performance and high light efficiency and have been attracting great attention in the field of high-power LEDs. Forming a p-type ohmic contact electrode with high reflectance and low ohmic contact resistance is the key to realize high-efficiency FCLEDs ${ }^{[5]-[7]}$. However, in the high power LED lighting market, automobile headlights, and searchlights require high current density and LED light extraction efficiency ${ }^{[8]-[10]}$. Meanwhile, automotive headlights are used in an array of dense packaging, requiring a higher LED vertical light efficiency ${ }^{[11]}$. Ag-based reflective p-electrodes are widely used in FCLEDs owing to their superior electrical properties and high reflectivity in the visible wavelength range ${ }^{[12]}$. However, the $\mathrm{Ag}$ contact suffers from poor adhesion, inferior ohmic behavior, and thermal instability such as migration. Therefore, it is necessary to maintain a sufficient distance between the Ag mirror and a PN junction in the LED chip design, and cover it with a TiW barrier diffusion layer ${ }^{[13],}{ }^{[14]}$, resulting in the loss of emission area and thus reducing the output efficiency. In addition to the normal Ag-base FC LED device structures, there are also DBR-base FC LED

${ }^{1}$ Institute of Semiconductor Science and Technology, South China Normal University, 55 Zhongshan Avenue, Tianhe District, Guangzhou 510631, China

${ }^{2}$ State Key Laboratory for Artificial Microstructure and Mesoscopic Physics, School of Physics, Peking University, Beijing, China, 100871

Correspondence and requests for materials should be addressed to Z.-Y. Guo(e-mail: guozy@scnu.edu.cn) 
device structure which use ITO and interdigitated metal contact for current spreading layers., but these structures bring out degradation of the optical performance, reliability. and lifetime of the optoelectronic device with the high power input due to the poor current spreading ability of the interdigitated metal contact ${ }^{[15]-[17]}$.

In this paper, we report the demonstration of an FCLED with a novel composite reflection structure (CRS-FCLED) that simultaneously improves the light output power (LOP) and vertical light extraction efficiency. Compared with the commonly used FCLED with a single Ag-mirror layer as the reflective layer, the CRS-FCLED with an $\mathrm{Ag} / \mathrm{SiO}_{2} /$ distributed Bragg reflector (DBR)/SiO 2 reflective structure exhibited improved reflectance. Further investigation proved that the $\mathrm{SiO}_{2} / \mathrm{DBR} / \mathrm{SiO}_{2}$ composite layer covers the sidewall and part of the area of the $\mathrm{n}-\mathrm{GaN}$ contact holes that are evenly distributed across the whole area of the LED chips, which improves the light extraction efficiency. When operated under currents of 700, 1000, and $1500 \mathrm{~mA}$, the light outputs of the CRS-FCLEDs are improved by 3.8, 5.1, and $6.3 \%$, respectively, and the corresponding external quantum efficiencies (EQEs) improved by 3.4, 4.7, and $6.0 \%$, respectively. Moreover, the light emission intensity distributions and far-field angular light emission measurements proved that the CRS-FCLED can strengthen the light output in the vertical direction, which is advantageous for high-power field applications, such as headlamps for automobiles.

\section{Results}

The LED samples were grown on the c-plane of the patterned sapphire substrate using the metal-organic chemical vapor deposition (MOCVD) method. The epitaxial structures of the LED from the bottom to the top consist of an AlN buffer layer with a thickness of $20 \mathrm{~nm}$, a 3- $\mu \mathrm{m}$ undoped GaN layer, a heavily Si high doping $\mathrm{n}-\mathrm{GaN}$ layer with a thickness of $2.5 \mu \mathrm{m}$, an InGaN/GaN superlattice structure with a thickness of $120 \mathrm{~nm}$ that can act as a strain release layer, five pairs of InGaN/GaN multiple quantum wells (MQWs) with a thickness of $30 \mathrm{~nm}$, a 40-nm low temperature p-GaN layer, a 48-nm p-AlGaN/GaN electron blocking layer, and a 110-nm Mg- doped p-GaN layer. The dimensions of the CRS-FCLED are $1400 \times 1400 \mu \mathrm{m}^{2}$ for high-power applications.

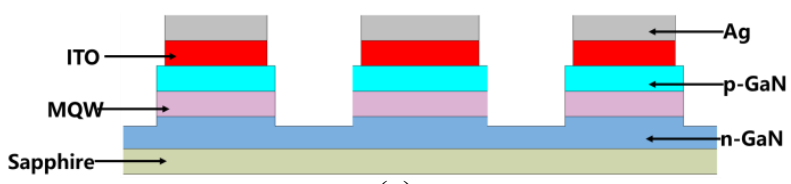

(a)

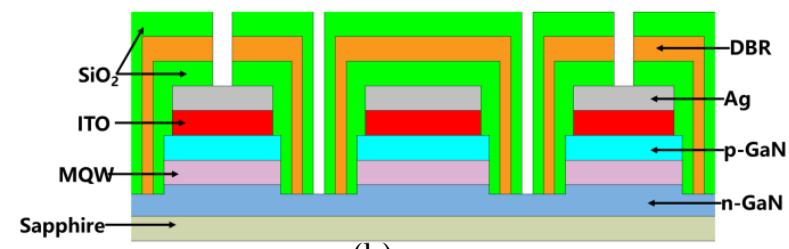

(b)

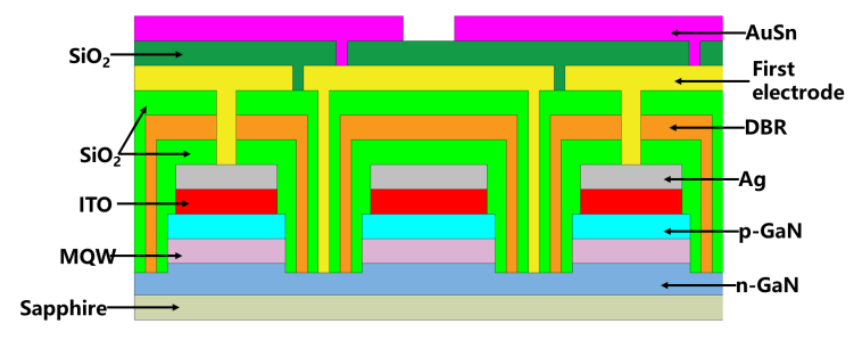

(c)

Fig. 1. Main fabrication process of the CRS-FCLED.

The main fabrication processes of the CRS-FCLED are shown in Fig. 1. The detailed processing steps are as follows: 
the $\mathrm{n}-\mathrm{GaN}$ contact holes are fabricated by an inductively coupled plasma (ICP) etching method with a $\mathrm{BCl}_{3} / \mathrm{Cl}_{2} \mathrm{mixture}$ gas. A 10-nm ITO contact layer was then deposited on the $\mathrm{p}-\mathrm{GaN}$ by the magnetron sputtering method, followed by annealing in $\mathrm{N}_{2}$ ambient at $550{ }^{\circ} \mathrm{C}$ to improve the contact properties. Subsequently, a layer of $\mathrm{Ag}(120 \mathrm{~nm})$ is sputtered on top of the ITO that acts as the reflecting layer [Fig. 1(a)]. For the fabrication of the CRS structure, a layer of $\mathrm{SiO}_{2}$ $(300 \mathrm{~nm}$ ) was first deposited on top of the Ag-mirror layer, after which an insulating DBR structure with 20 pairs of periodically arranged $\mathrm{SiO}_{2}$ and $\mathrm{TiO}_{2}$ layers $(82.3 \mathrm{~nm} / 40.5 \mathrm{~nm})$ is deposited on the top of the $\mathrm{SiO}_{2}$ layer and partially filled the via holes. Then, a $300-\mathrm{nm} \mathrm{SiO}_{2}$ layer is deposited on top of the DBR structure for better passivation protection. Finally, the p-GaN contact holes and $\mathrm{n}-\mathrm{GaN}$ contact holes were fabricated by ICP etching with a $\mathrm{CF}_{4} / \mathrm{O}_{2}$ gas mixture as the etching gas source [Fig. 1(b)]. After the fabrication of the CRS structure, the first electrode layers of $\mathrm{Cr}(0.5$ $\mathrm{nm}) / \mathrm{Al}(1 \mu \mathrm{m}) / \mathrm{Cr}(40 \mathrm{~nm}) / \mathrm{Pt}(0.2 \mu \mathrm{m})$ were deposited on the DBR and filled the p-contact hole and $\mathrm{n}$-contact holes. $\mathrm{A} \mathrm{SiO}_{2}$ insulating layer with a thickness of $1.2 \mu \mathrm{m}$ is deposited on the top of the first electrode layer grown by plasmaenhanced chemical vapor deposition, with the interconnected holes formed by the buffer oxide etchant (BOE) wet etching method. Finally, an AuSn alloy solder layer was deposited by thermal evaporation to meet the welding reliability requirements for headlamp applications [Fig. 1(c)].

The p-type ohmic contact electrodes of FCLEDs should have high reflectivity and low contact resistance. To realize this, metallic and DBR mirrors can be used as highly reflective layers in flip chips owing to their high reflectance in the visible wavelength range ${ }^{[18]}$. Further, ITO was sandwiched between the reflection layer and the p-GaN to decrease the p-type contact resistance. on the other hand, DBR instead of metallic mirrors as the same reflective properties ${ }^{[19]}$. For comparing the reflectance, $\mathrm{Ni}(0.1 \mathrm{~nm}) / \mathrm{Ag}(150 \mathrm{~nm})$, ITO $(10 \mathrm{~nm}) / \mathrm{Ag}(150 \mathrm{~nm})$, ITO $(10 \mathrm{~nm}) / \mathrm{DBR}(2.1 \mu \mathrm{m})$, and ITO $(10 \mathrm{~nm}) / \mathrm{Ag}(150 \mathrm{~nm}) / \mathrm{SiO}_{2}(300 \mathrm{~nm}) / \mathrm{DBR}(2.1 \mu \mathrm{m}) / \mathrm{SiO}_{2}(300 \mathrm{~nm})$ films were deposited on the quartz glass. To simulate the actual reflection of the chip, all the layers are patterned by a photoresist mask and etched by ICP etching with a $\mathrm{CF}_{4} / \mathrm{O}_{2}$ gas mixture. The reflectance as a function of wavelength is shown in Fig 2.

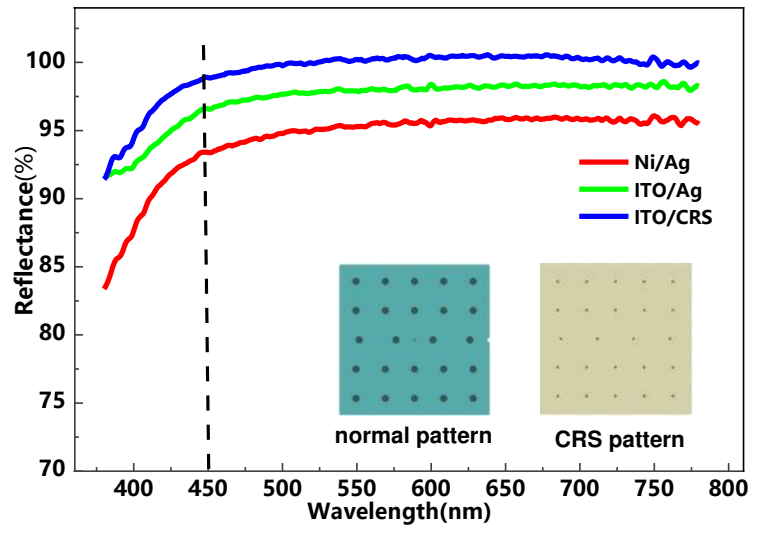

Fig. 2. Reflectances of Ni/Ag, ITO/Ag, and ITO/CRS films

At the wavelength of $450 \mathrm{~nm}$, the measured reflectances of $\mathrm{Ni} / \mathrm{Ag}$, ITO/Ag, and ITO/CRS are $93.3 \%$, 96.5\%, and $98.8 \%$, respectively. Ni/Ag exhibits the lowest reflectance due to strong absorption of light by the underlying Ni layer ${ }^{[14]}$. Further, the higher reflectance of ITO/CRS films in visible light than that of ITO/Ag films indicates that ITO/CRS films can efficiently substitute the normal ITO-Ag reflection systems.

Fig. 3(a,b) shows a top-view scanning electron microscope (SEM) image of the normal Ag-based FCLED and CRSFCLED, respectively. Their layouts are nearly identical except for the reflective layer structure. The $\mathrm{n}$-contact holes are evenly distributed over the entire area of the LED chip to enhance current spreading. The cross-sectional SEM images of both LEDs are shown in Fig. 3(c,d), and the corresponding schematic illustrations are shown in Fig. 3(e,f). As shown in Fig. 3(c,e), the diameter of the $\mathrm{n}$-contact holes in the Ag layer is larger than that of the $\mathrm{n}$-GaN contact holes in the normal Ag-based FCLEDs, to avoid Ag migration on forward current aging or bulk leakage by electrode destruction near the V-pit defect region ${ }^{[20]}$. However, this diameter mismatch can form an emission loss area. The light emitted from the sidewall of each n-GaN contact hole can be absorbed by n-pad metals with low reflectance, such as $\mathrm{Cr}$ and $\mathrm{Au}$, which can decrease the light extraction efficiency. This emission loss phenomenon can be effectively reduced in CRS-FCLEDs. As shown in Fig. 3(d,f), at the edge of the Ag mirror, the DBR mirror continues to extend the reflection area, covering the upper and lower mesa gentle slope and the n-via area to maximize the reflection area. As a result, CRS-FCLEDs can achieve improved reflection performance compared with normal Agbased FCLEDs. 


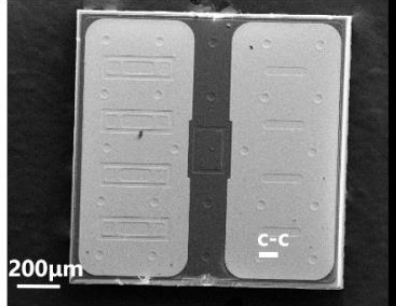

(a)

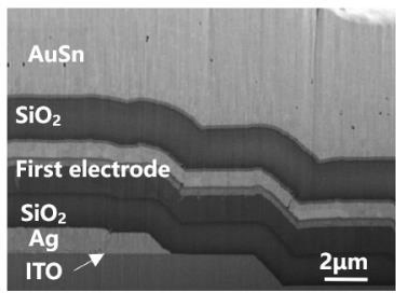

(c)

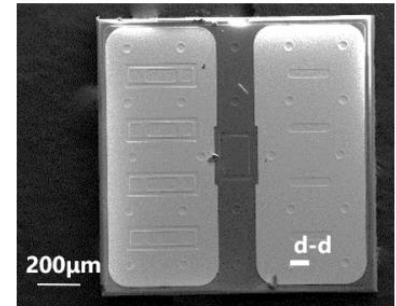

(b)

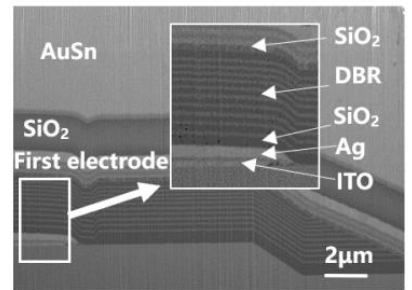

(d)

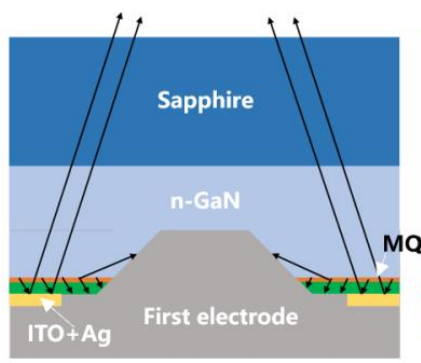

(e)

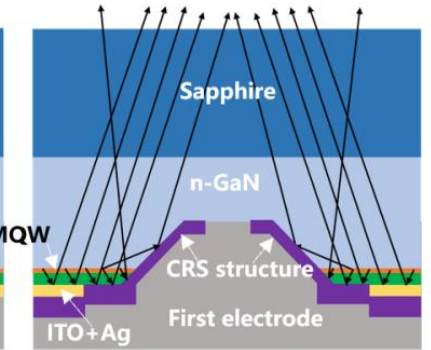

(f)

Fig. 3. (a) Top-view SEM image of normal Ag-based FCLED. (b) Top-view SEM image of CRS-FCLED. (c) Cross-sectional SEM image of normal Ag-based FCLED milled by FIB along c-c direction. (d) Cross-sectional SEM image of CRS-FCLED milled by FIB along the d-d direction. (e) Schematic illustration of the light extraction of normal Ag-based FCLEDs. (f) Schematic illustration of the light extraction of CRS-FCLEDs.

The dependence of the forward voltage and LOP versus injection current for the normal Ag-based FCLED and CRS-FCLED are shown in Fig. 4(a). The I-V curves of the two LEDs are nearly identical, indicating that both have similar p-type and n-type contact spreading resistance ${ }^{[21]}$. As the injection current increased, the output power of the CRS-FCLEDs showed better performance. At $350 \mathrm{~mA}$, the LOP of the CRS-FCLED is $3.8 \%$ higher, which is further increased to $5.1 \%$ at $1000 \mathrm{~mA}$. Finally, at $1500 \mathrm{~mA}$, the LOP of the CRS-FCLED is $6.3 \%$ higher than that of the normal Ag-based FCLED. Meanwhile, the EQE of the CRS-FCLEDs improved by 3.4, 4.7, and $6.0 \%$ at 350, 1000, and $1500 \mathrm{~mA}$, respectively [Fig. 4(b)]. The improvements can be attributed to the increased high reflection area, which helps to increase the light extraction efficiency. In addition, the degree of enhancement is larger at high injection currents, which can be explained by the better protection effect of the $\mathrm{SiO}_{2} / \mathrm{DBR} / \mathrm{SiO}_{2}$ passivation layer that hindered $\mathrm{Ag}$ migration to the GaN material degradation with a large current density, which improves the EQE.
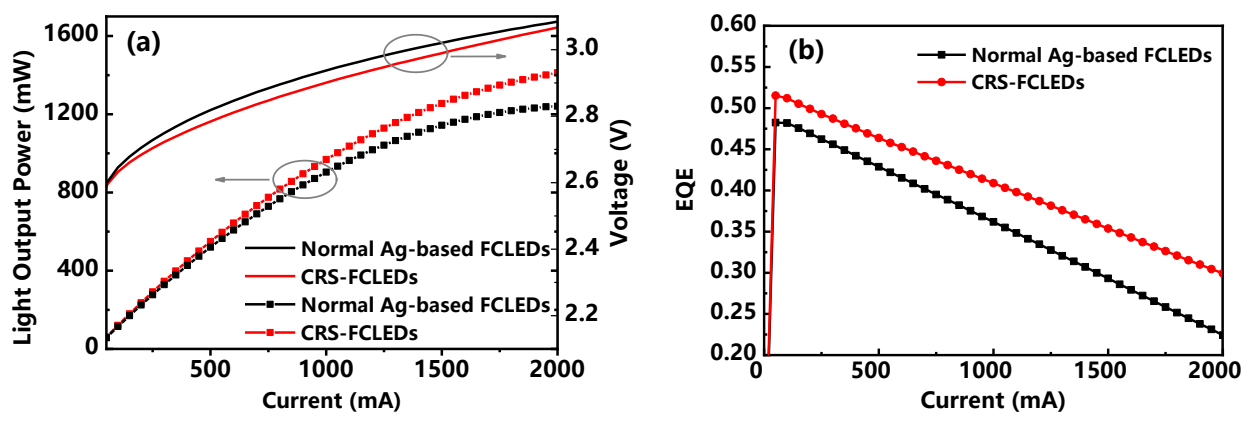

Fig. 4. (a) Forward voltage and LOP versus injection current for normal Ag-based FCLED and CRS-FCLED. (b) EQE versus injection current for normal Ag-based FCLED and CRS-FCLED. 
Current crowding across the thick p reflective electrode adjacent to the n-electrode would result in surface leakage and output drop because of Ag migration on forward current aging ${ }^{[22]}$; therefore, Ag migration protection will determine the reliability and $\mathrm{EQE}$ of the flip-chip in high current injection conditions. In contrast with the normal Agbased FCLED with a $\mathrm{SiO}_{2}$ passivation layer that is less than $1 \mu \mathrm{m}$ thick, the CRS structure covers the p reflective electrode and n-electrode with over 3- $\mu \mathrm{m} \mathrm{SiO} / 2 / \mathrm{DBR} / \mathrm{SiO}_{2}$ sandwich passivation layer, which further effectively blocks the path of Ag migration to the n-electrode.

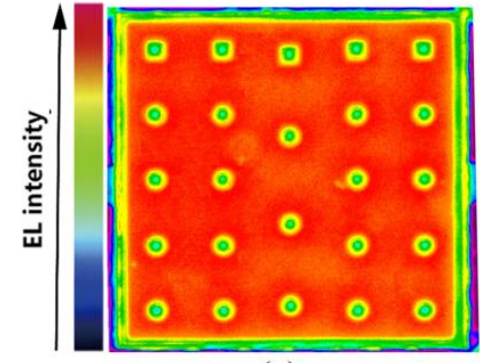

(a)

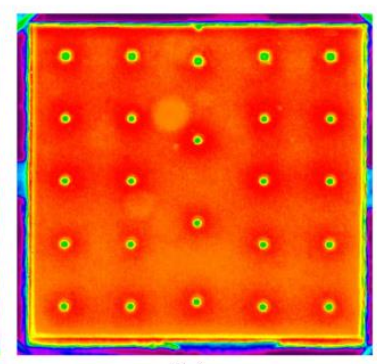

(b)

Fig. 5. (a) Light emission distributions in the normal Ag-based FCLED. (b) Light emission intensity distributions in the CRS-FCLED.

The light emission intensity distributions of the normal Ag-based FCLEDs and CRS-FCLEDs, when driven by an injection current of $1500 \mathrm{~mA}$ [Fig. 5], shows that the total emission region of the CRS-FCLEDs is larger, owing to the increased reflective area at the sidewall and partial area of the $\mathrm{n}-\mathrm{GaN}$ contact holes. Moreover, the emission intensity in the emission region around the $\mathrm{n}-\mathrm{GaN}$ contact holes is explicitly higher than that in other emission regions. It has been reported that the number of photons generated in the active region around the electrode is significantly higher than that in other regions of the LED because of the current crowding effect around the electrode ${ }^{[23]}$. Therefore, even though the increased total reflective area of the CRS-FCLEDs is negligible, the light extraction efficiency can be significantly improved because the number of photons generated around the electrodes is considerably higher than that in other emission regions.

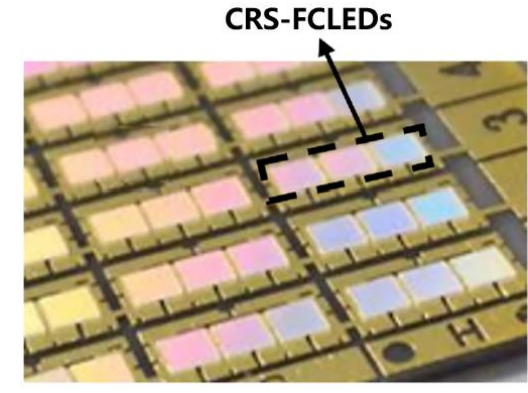

(a)

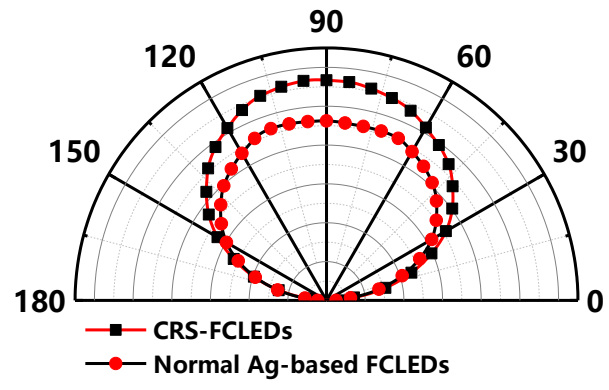

(b)

Fig. 6. (a) LED headlight module with dense matrix package. (b) Far-field radiation pattern of CRS-FCLED and normal Ag-based FCLED at $1500 \mathrm{~mA}$.

Fig. 6(a) shows the LED module fabricated with our CRS-FCLEDs for use in a headlight. All the LED chips were packaged in an AlN ceramic matrix with high thermal conductivity. For headlight applications, the LED module must have a high packaging density to obtain the maximized optical density per unit area, with the distance between LED modules designed to be minimized within a short range of only 100-200 $\mu \mathrm{m}$. However, high losses occur at large emission angles due to the narrow distance between chips, and this is not beneficial for the light output of the LED module. Fig. 6(b) shows the far-field angular light emission patterns of the normal Ag-based FCLEDs and CRSFCLEDs. The operational current is $1500 \mathrm{~mA}$ for both LEDs, which is in accordance with the normal working conditions of the headlight module. Compared with the normal Ag-based FCLEDs, the intensity of the emission light from the CRS-FCLEDs is significantly increased, especially in the vertical direction, and the intensity of the emission light in the large angle direction is rarely increased. This result can be well explained by the additional reflection area of the CRS-FCLEDs. As the location of the additional reflective area is on the sidewall and covers part of the $n-G a N$ contact holes, the photons emitted from the side wall can be extracted with the emission angle changing in the vertical 
direction. As a result, the CRS-FCLED with the unique characteristic of strengthened light output in the vertical direction is suitable for use in a headlamp.

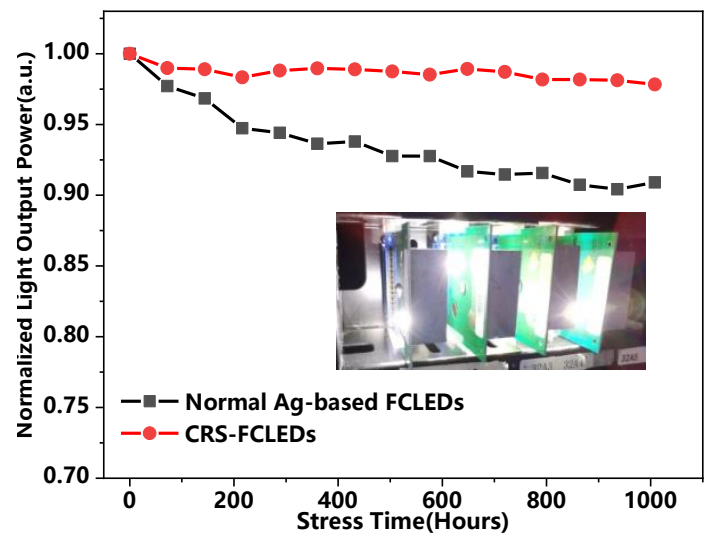

Fig. 7. Optical degradation of CRS-FCLED and normal Ag-based FCLED during high temperature operation life test.

Moreover, as shown in Fig. 7, the optical degradations of the CRS-FCLED and normal Ag-based FCLED at $85{ }^{\circ} \mathrm{C}$ were also investigated using an injection current of $1500 \mathrm{~mA}$. After high temperature operation life test $(1000 \mathrm{~h})$, the light output power of CRS-FCLED decreased by $3.07 \%$, whereas that of the normal Ag-based FCLED decreased by 9.92\%. Clearly, the CRS-FCLED exhibits significantly smaller optical degradation and thus, offers a higher device reliability as compared to the normal Ag-based FCLED. Further, the forward current aging resulted in a noticeable output degradation in Ag-based FCLEDs due to the surface migration of $\mathrm{Ag}{ }^{[24]}$. The CRS structure covered by the $\mathrm{SiO}_{2} / \mathrm{DBR} / \mathrm{SiO}_{2}$ passivation layers consisting of three dielectric stack layers is found to be effective in suppressing the $\mathrm{Ag}$ migration. In addition, the mentioned structure is considerably effective in passivating the exposed surfaces of ITO and $\mathrm{n}-\mathrm{GaN}$ layers after ICP etching, resulting in decreasing the trap density near the surface, minimizing the leakage current through the surface of the LED.

\section{Discussion}

In summary, FCLEDs with a novel composite reflection structure of $\mathrm{Ag} / \mathrm{SiO}_{2} / \mathrm{DBR} / \mathrm{SiO}_{2}$ were fabricated, which simultaneously improved the light extraction efficiency and light output in the vertical direction. Compared with the conventional FCLEDs with a single Ag mirror as the reflective layer, the reflective area of the CRS-FCLEDs is increased because the sidewall and part of the $\mathrm{n}-\mathrm{GaN}$ contact holes had been covered by the highly reflective $\mathrm{SiO}_{2} / \mathrm{DBR} / \mathrm{SiO}_{2}$ sandwich structure. As a result, the LOP of the CRS-FCLEDs increased by $6.3 \%$ at an operational current of $1500 \mathrm{~mA}$, while the corresponding EQE was improved by $6.0 \%$, and it exhibited markedly smaller optical degradation and thus higher device reliability as compared to t normal Ag-based FCLED. Moreover, the light emission intensity distributions and the far-field angular light emission pattern proved that it exhibited a higher light output in the vertical direction, suggesting that the CRS-FCLEDs have potential in headlamp lighting.

\section{Method}

Epitaxial growth. The LED samples were grown on the c-plane of the patterned sapphire substrate using the metalorganic chemical vapor deposition (MOCVD) method. Trimethylgallium (TMGa), trimethylindium (TMIn), trimethylaluminum (TMAl) and ammonia $\left(\mathrm{NH}_{3}\right)$ were used as gallium $(\mathrm{Ga})$, indium $(\mathrm{In})$, aluminum (Al) and nitrogen (N) sources material, respectively. Silane $\left(\mathrm{SiH}_{4}\right)$ and biscyclopentadienyl magnesium $\left(\mathrm{CP}_{2} \mathrm{Mg}\right)$ were used as the $\mathrm{n}$-type doping and p-type doping sources, respectively.

Optical characterization. The surface morphologies of the LED structures were detected by using a top-view scanning electron microscope (ZEISS $\Sigma$ IGMA). The reflectance as a function of wavelength was measured using a Spectrophotometer (HITACHI U-3900). Forward voltage and LOP versus injection current were measured by integrating sphere (EVERFINE HAAS-3000). The light emission distributions of both LED structures were obtained by thermal Imaging Spectrometer (OPHIR Photonics spiricon). Far-field radiation pattern of the LED samples were measured through spatial spectral distribution test system (EVERFINE GO-SPEX100). The optical degradations of LED samples were measured by using (EVERFINE LT-300A) accelerated aging and life test system. 


\section{References}

[1] J. Bhardwaj, J. M. Cesaratto, I. H. Wildeson, H. Choy, A. Tandon, W. A. Soer, P. J. Schmidt, B. Spinger, P. Deb, O. B. Shchekin, and W. Götz. Progress in high-luminance LED technology for solid-state lighting. Phys. Status Solidi A 214 ,1600826 (2017). DOI: 10.1002/pssa.201600826.

[2] S. P. Ying and W. B. Shen. Thermal analysis of high-power multichip COB light-emitting diodes with different chip sizes, IEEE Trans. Electron Devices 62, 896-901 (2015). DOI: 10.1109/TED.2015.2390255.

[3] X. H. Tao. Performance Characterization and Theoretical Modeling of Emitted Optical Power for High-Power White-LED Devices. IEEE Trans. Electron Devices 62, 1511-1515 (2015). DOI: 10.1109/TED.2015.2410032.

[4] C.-M. Yang, D.-S. Kim, S.-G. Lee, J.-H. Lee, Y. S. Lee, and J.-H. Lee. improvement in electrical and optical performances of GaN-based LED with $\mathrm{SiO}_{2} / \mathrm{Al}_{2} \mathrm{O}_{3}$ double dielectric stack layer. IEEE Electron Device Lett 33, 564-566 (2012). DOI: 10.1109/LED.2012.2185675.

[5] G. J. Lee, I. Y. Hong, T. K. Kim, H. J. Park, S. K. Oh, Y.-J. Cha, M. J. Park, K. J. Choi, and J. S. Kwak. Design of ITO/SiO $/ / \mathrm{TiO}_{2}$ distributed Bragg reflectors as a p-type electrode in GaN-based flip-chip light emitting diodes. Appl. Surf. Sci 477, 220-225 (2019). DOI: 10.1016/j.apsusc.2017.10.144

[6] W. C. Chong and K. M. Lau. Performance Enhancements of Flip-Chip Light-Emitting Diodes With High-Density n-Type Point-Contacts. IEEE Electron Device Lett 35, 1049-1051 (2014). DOI: 10.1109/LED.2014.2349956.

[7] S. Zhou, C. Zheng, J. Lv, Y. Gao, R. Wang, and S. Liu. GaN-based flip-chip LEDs with highly reflective ITO/DBR p-type and via hole-based n-type contacts for enhanced current spreading and light extraction. Opt. Laser Technol 92, 95-100 (2017). DOI: 10.1016/j.optlastec.2017.01.017.

[8] Z. T. LI, Y Tang, J Li, X.R. Ding, C.M, Yan and B. H. Yu. Effect of flip-chip height on the optical performance of conformal white-lightemitting diodes. Optics Letters 43, 1015-1018 (2018). DOI: 10.1364/OL.43.001015.

[9] N. Liu, X. Yi, L. Wang, X. Sun, L. Liu, Z. Liu, J. Wang, and J. Li. Light extraction improvement of blue light-emitting diodes with a metaldistributed Bragg reflector current blocking layer. Appl. Phys. A 118, 863-867, (2015).

[10] X. R. Ding, Y. Tang, Z.T. Li, J. S. Li, Y. X. Xie, and L.W. Lin. Multichip LED Modules With V-Groove Surfaces for Light Extraction Efficiency Enhancements Considering Roughness Scattering. IEEE Trans. Electron Devices 64, 182-188 (2017). DOI: 10.1109/ted.2016.2628788

[11] J.-S. Park, J. Han, J.-W. Han, H. Seo, J.-T. Oh, T.-Y. Seong. Improving the output power of near-ultraviolet InGaN/GaN-based light emitting diodes by enhancing the thermal and electrical properties of Ag-based reflector. Superlattices Microstructures 64, 7-14 (2013). DOI: 10.1016/j.spmi.2013.09.019

[12] J.-H. Lee, S.-M. Hwang, N.-S. Kim, and J.-H. Lee. InGaN-based high-power flip-chip LEDs with deep-hole-patterned sapphire substrate by laser direct beam drilling. IEEE Electron Device Lett 31, no. 7 (2010). DOI: 10.1109/LED.2010.2048887.

[13] J. O. Song, J. S. Kwak, Y. Park, and T.-Y. Seong. Ohmic and degradation mechanisms of Ag contacts on p-type GaN. Appl. Phys. Lett 86, 062104 (2005). DOI: 10.1063/1.1863441.

[14] J. H. Son, Y. H. Song, H. K. Yu, and J.-L. Lee. Effects of Ni cladding layers on suppression of Ag agglomeration in Ag-based Ohmic contacts on p-GaN. Appl. Phys. Lett. 95, 062108 (2009). DOI: 10.1063/1.3206742.

[15] S Zhou, X Liu, H Yan, Z Chen, S Liu. Highly efficient GaN-based high-power flip-chip light-emitting diodes. Optics Express 27, A669-A692 (2019). DOI: 10.1364/OE.27.00A669

[16] S. J. Zhou, X. T. Liu, Y. Gao, Y. Liu, M. L. Liu, Z. Y. Liu, C. Q. Gui, and S. Liu. Numerical and experimental investigation of GaN-based flip-chip light-emitting diodes with highly reflective Ag/TiW and ITO/DBR Ohmic contacts. Optics Express 25, 26615-26627 (2017). DOI: 10.1364/OE.25.026615.

[17] G. Y. Shiu, K. T. Chen, F. H. Fan, K. P. Huang, W. J. Hsu, J. J. Dai, C. F. Lai and C. F. Lin. InGaN Light-Emitting Diodes with an Embedded Nanoporous GaN Distributed Bragg Reflectors. Sci Rep 6, 29138 (2016). DOI: 10.1038/srep29138.

[18] T. H. Lin, S. J. Wang, Y. C. Tu, C. H. Hung, T.H. Yu. Improving the performance of power GaN-based thin-film flip-chip LEDs through a two fold roughened surface. Materials Science in Semiconductor Processing 45, 69-75 (2016). DOI: 10.1016/j.mssp.2016.01.010.

[19] M. Genç, V. Sheremet, et al. Distributed contact flip chip InGaN/GaN blue LED; comparison with conventional LEDs. Superlattices and Microstructures 128, 9-13 (2019). DOI: 10.1016/j.spmi.2019.01.008.

[20] S. Jeong, M. S. Kim, S.-N. Lee, and H. Kim. Forward and reverse current aging of GaN-based light-emitting diodes fabricated with Ag-based reflective electrodes. Mat. Sci. Semicon. Proc 90, 72-77 (2019). DOI: 10.1016/j.mssp.2018.10.005.

[21] H. T. Chen, Y. F. Cheung, H. W. Choi, S. C. Tan, and S. Y. Hui. Reduction of thermal resistance and optical power loss using thin-film lightemitting diode (LED) structure. IEEE Trans. Ind. Electron 62, 6925-6933 (2015). DOI: 10.1109/TIE.2015.2443106.

[22] J. K. Sheu, I-H. Hung, W. C. Lai, S. C. Shei, and M. L. Lee. Enhancement in output power of blue gallium nitride-based light-emitting diodes with omnidirectional metal reflector under electrode pads. Appl. Phys. Lett 93, 103507 (2008). DOI: 10.1063/1.2980422.

[23] H. Kim, J. Cho, Y. Park, T. Y. Seong. Leakage current origins and passivation effect of GaN-based light emitting diodes fabricated with Ag p-contacts. Appl. Phys. Lett 92, 092115(2008). DOI: 10.1063/1.2844887.

[24] X. Liu, N. Li, J.F. Hu, Y. L. Gao, R. Q. Wang and S.G. Zhou. Comparative Study of Highly Reflective ITO/DBR and Ni/Ag ohmic Contacts for GaN-Based Flip-Chip Light-Emitting Diodes. ECS J. Solid State Sci. Technol 7, Q116-Q122 (2018). DOI: 10.1149/2.0191806jss.

\section{Acknowledgements}

This work was supported in part by the Key-Area Research and Development Program of Guangdong Province (2019B010128002, 2020B010171002), Foshan Science and Technology Bureau (1920001000724), Guangdong Science and Technology Project (Grant 2019KTSCX084), and Guangzhou Science and Technology Program Key Projects (201807010083).

\section{Author Contributions}

L.X. conceived the experiments, L.X. carried out optical analysis, SEM of the LED samples, and J.L.Z. performed the Far-field radiation and Light emission distributions measurement and analysis, H.Q.S contributed the calculation result of reliability . Z.Z.C. and Z.Y.G. reviewed the manuscript.

\section{Additional Information}

Competing Interests: The authors declare that they have no competing interests. 
Figures

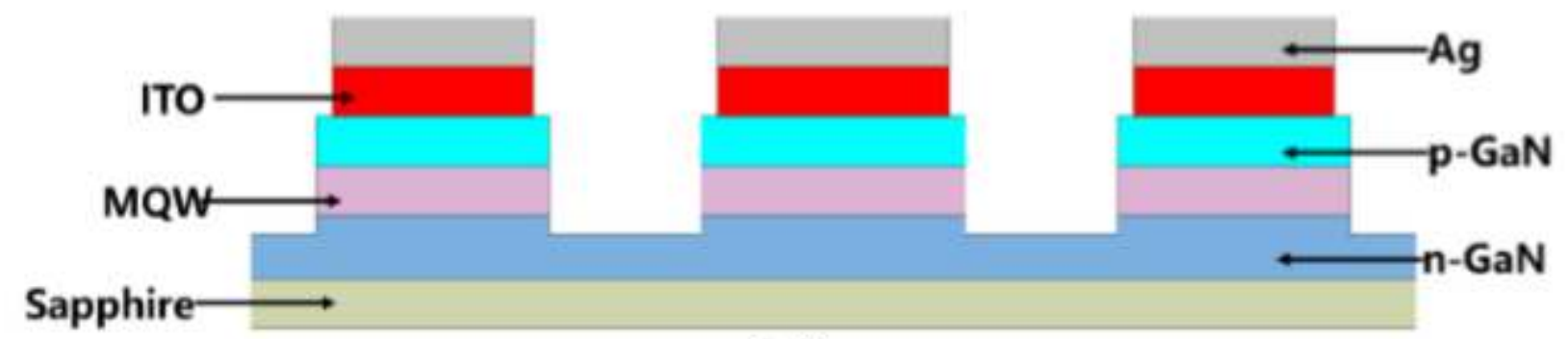

(a)

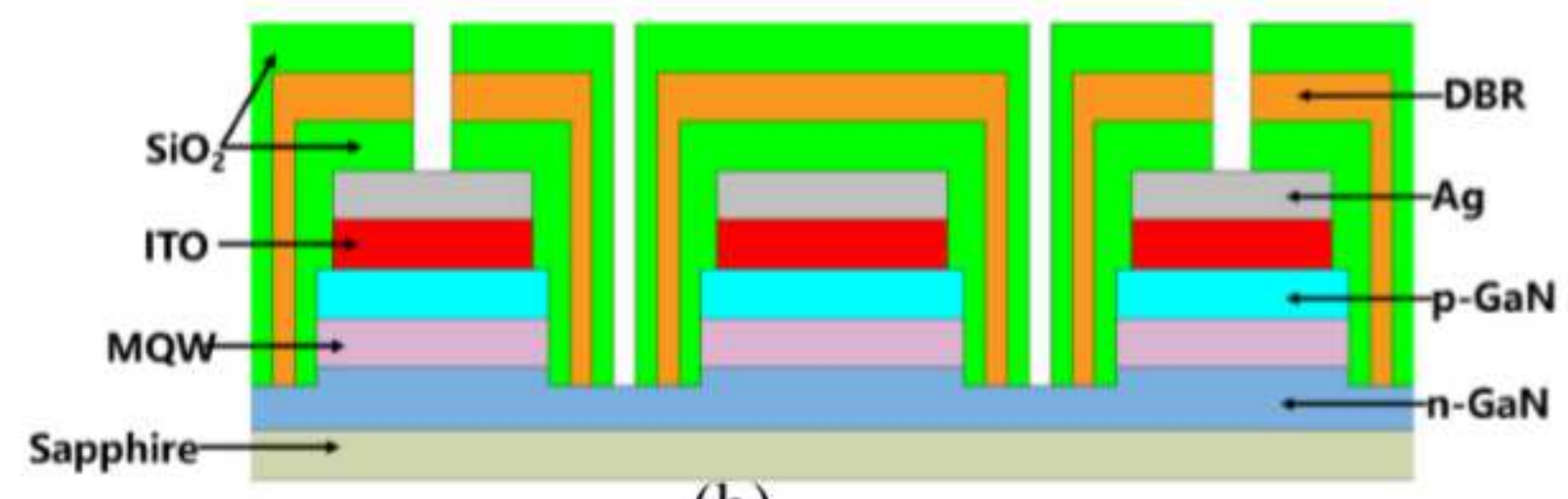

(b)

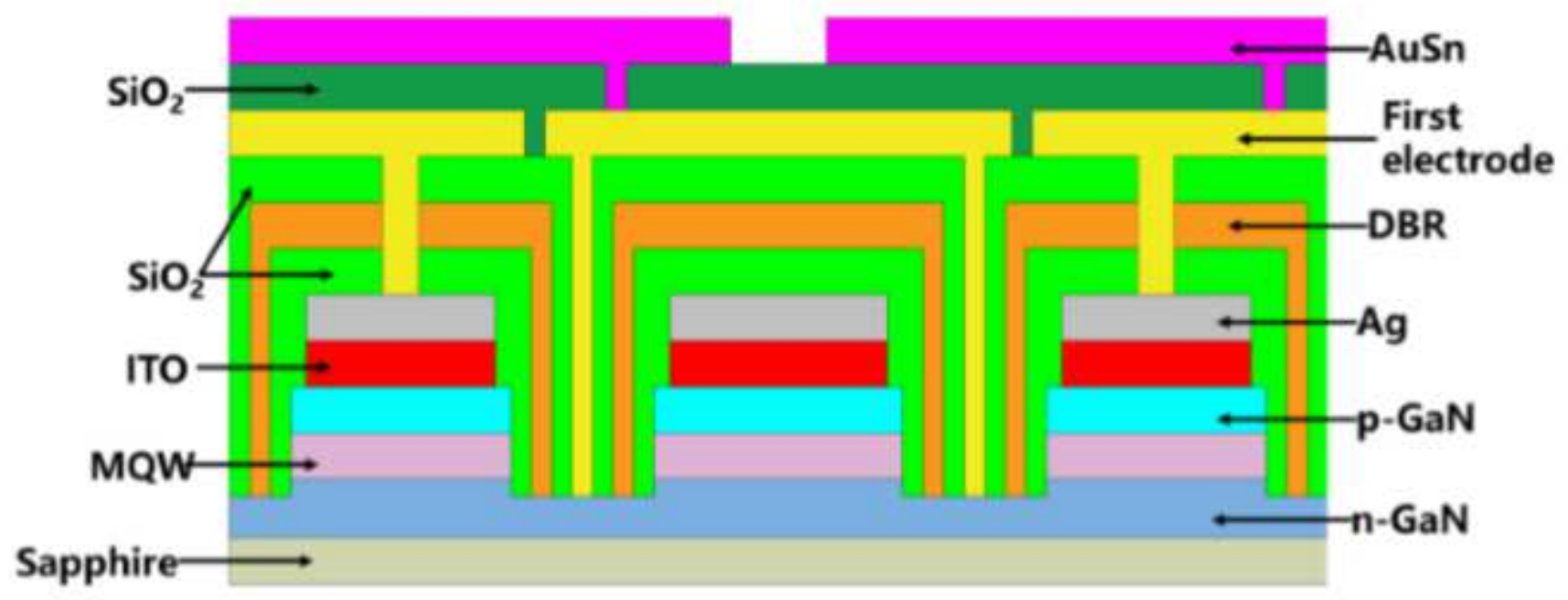

(c)

Figure 1

Main fabrication process of the CRS-FCLED. 


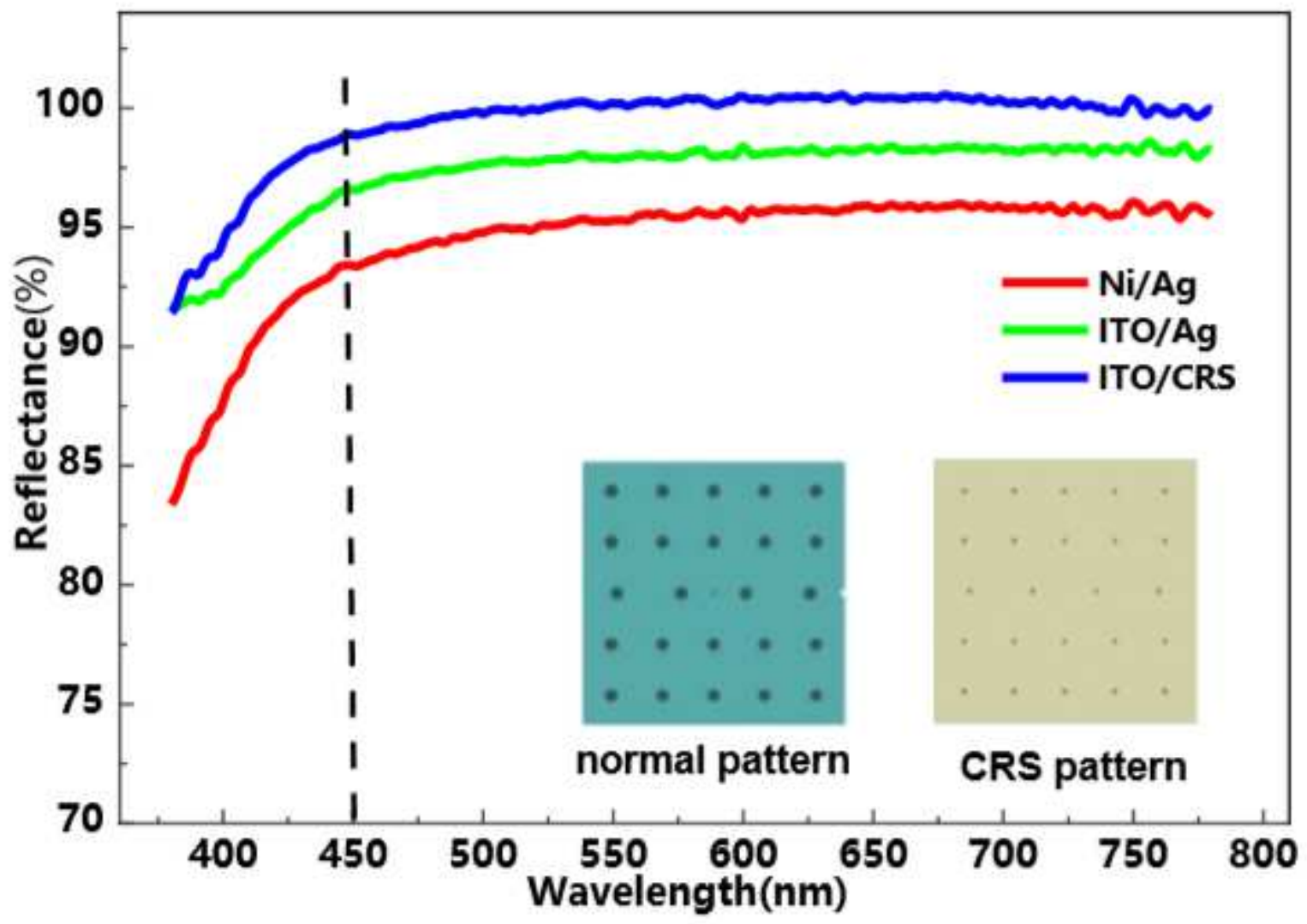

Figure 2

Reflectances of $\mathrm{Ni} / \mathrm{Ag}, \mathrm{ITO} / \mathrm{Ag}$, and ITO/CRS films 


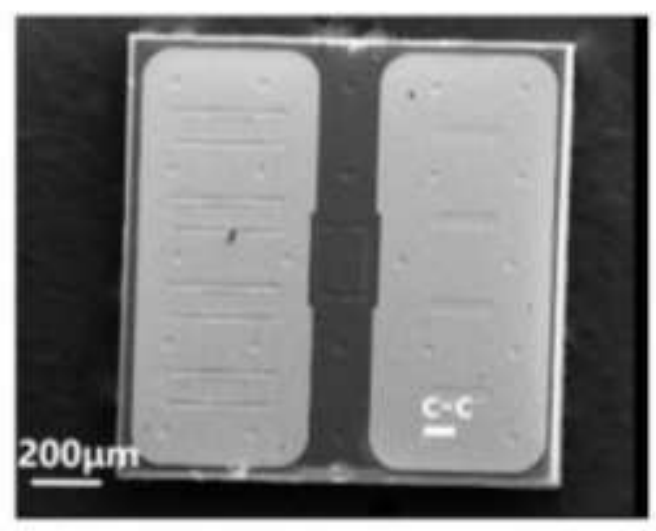

(a)

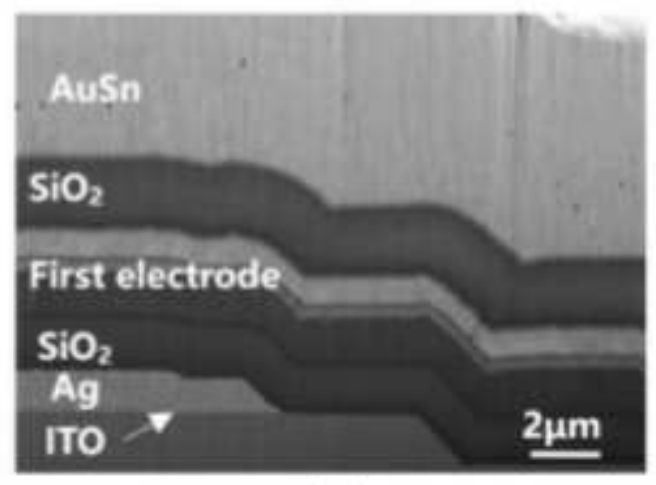

(c)

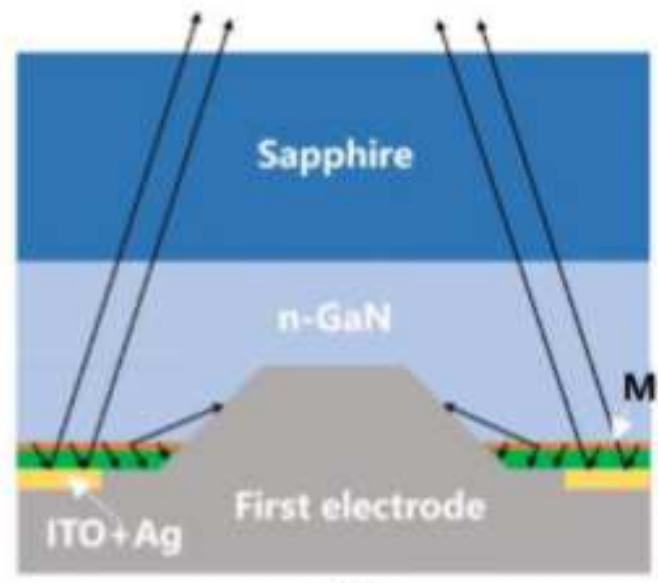

(e) (b)

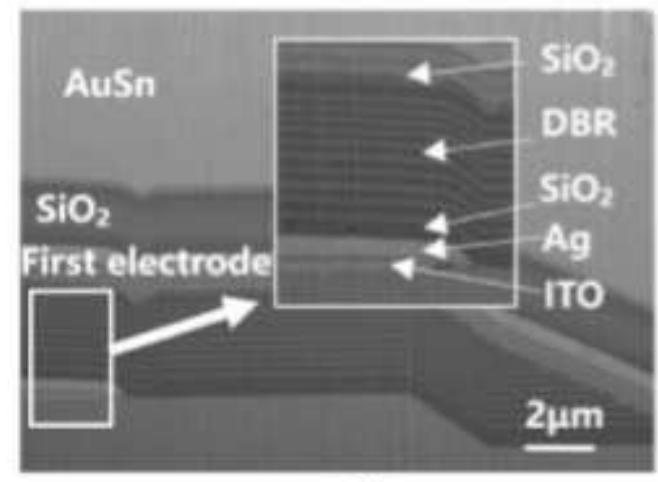

(d)
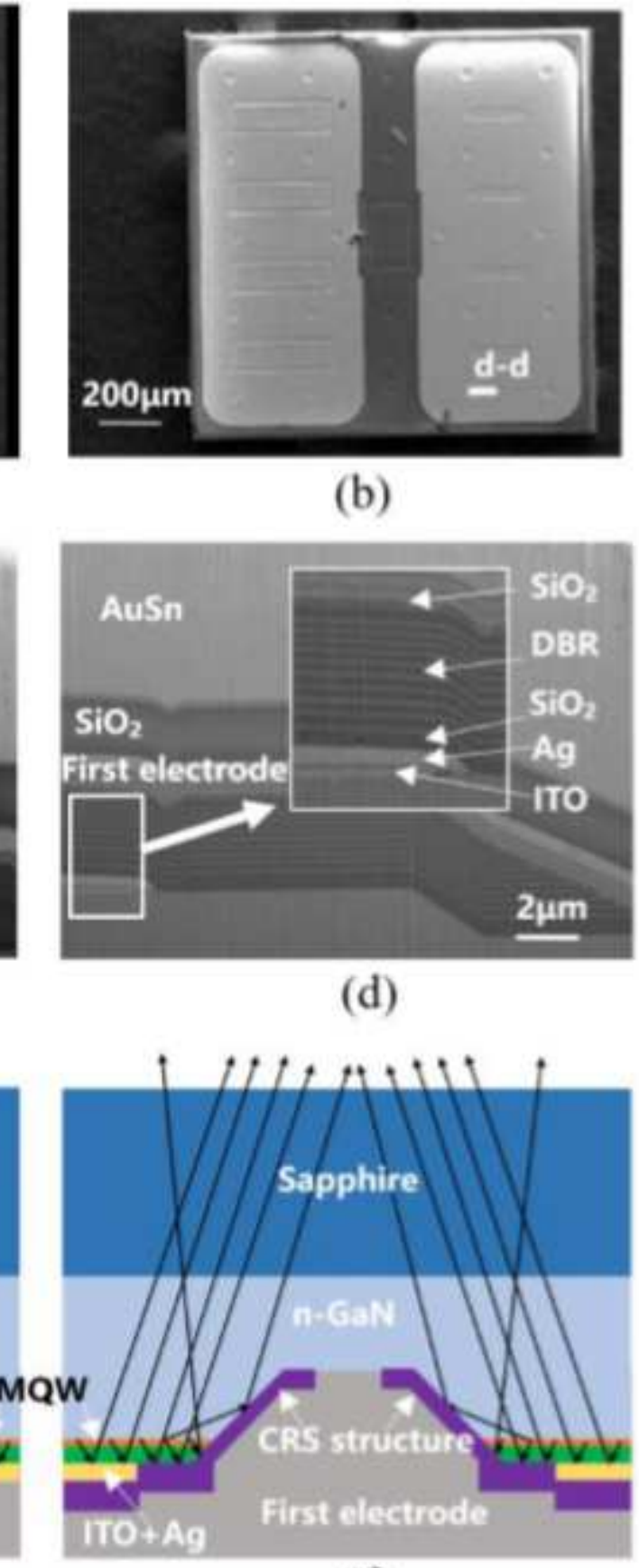

(f)

Figure 3

(a) Top-view SEM image of normal Ag-based FCLED. (b) Top-view SEM image of CRS-FCLED. (c) Crosssectional SEM image of normal Ag-based FCLED milled by FIB along c-c direction. (d) Cross-sectional SEM image of CRS-FCLED milled by FIB along the d-d direction. (e) Schematic illustration of the light extraction of normal Ag-based FCLEDs. (f) Schematic illustration of the light extraction of CRS-FCLEDs. 

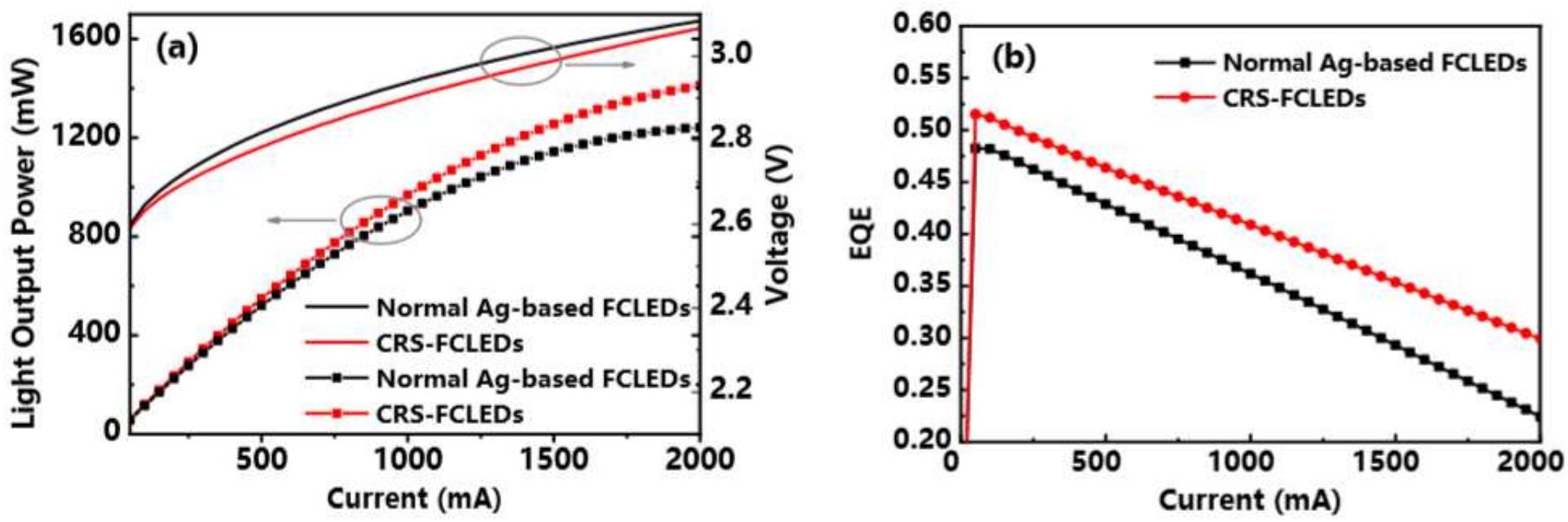

Figure 4

(a) Forward voltage and LOP versus injection current for normal Ag-based FCLED and CRS-FCLED. (b) EQE versus injection current for normal Ag-based FCLED and CRS-FCLED.

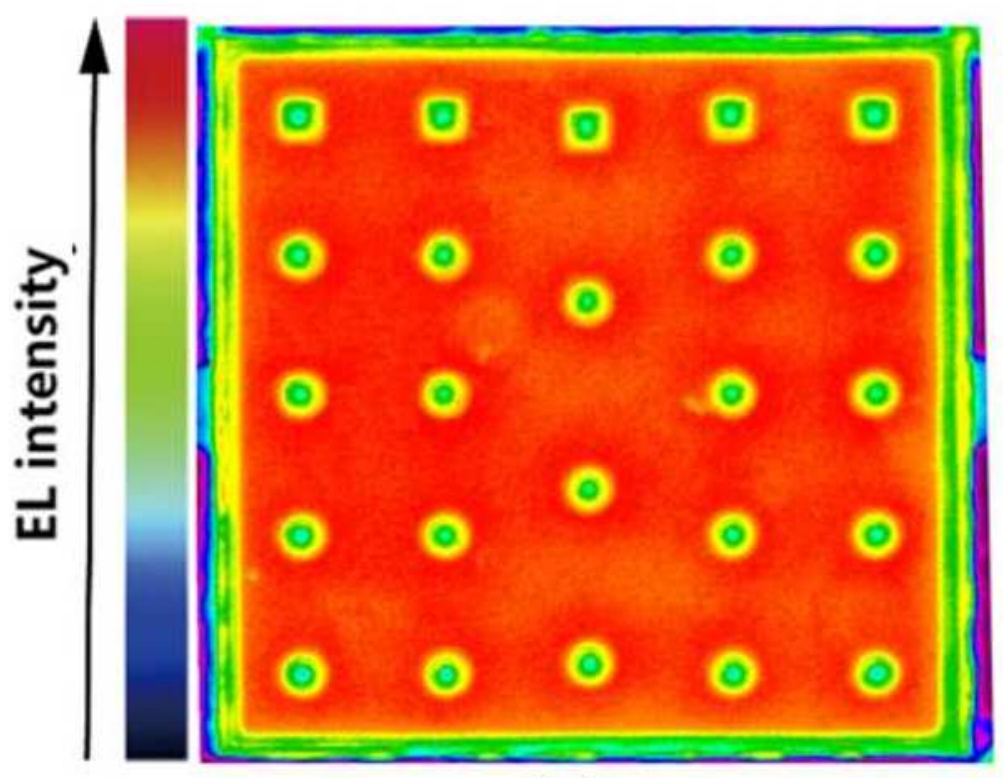

(a)

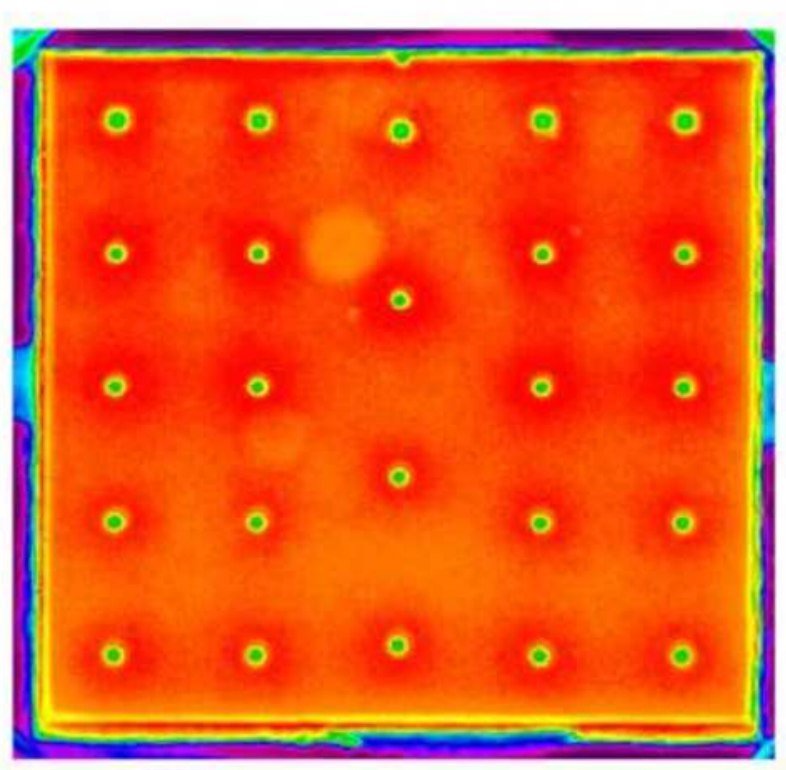

(b)

Figure 5

(a) Light emission distributions in the normal Ag-based FCLED. (b) Light emission intensity distributions in the CRS-FCLED. 


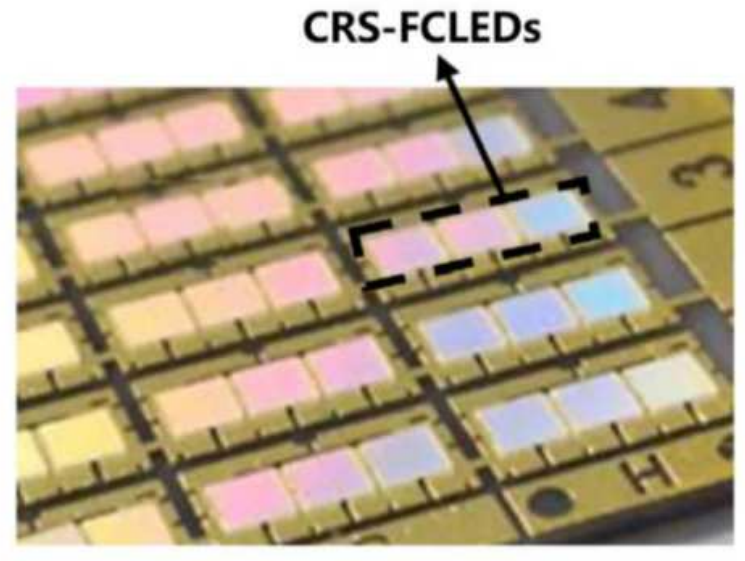

(a)

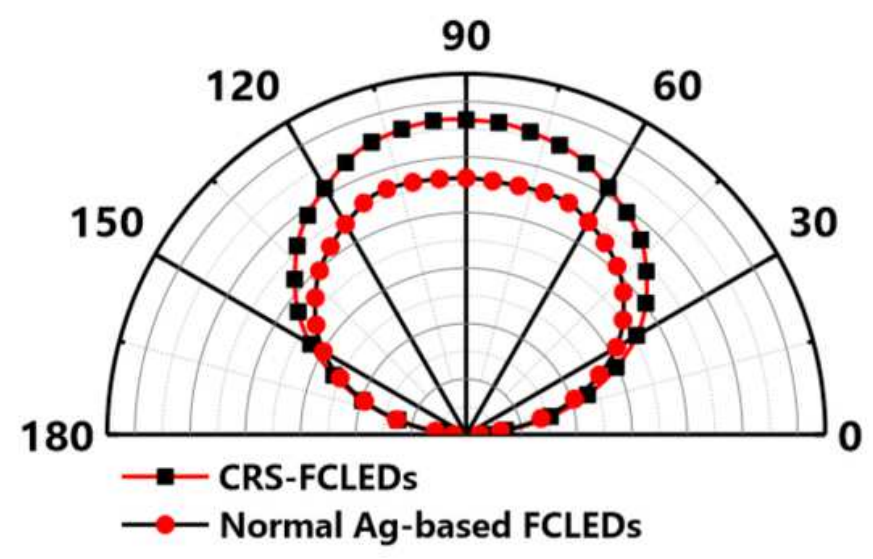

(b)

Figure 6

(a) LED headlight module with dense matrix package. (b) Far-field radiation pattern of CRS-FCLED and normal Ag-based FCLED at $1500 \mathrm{~mA}$

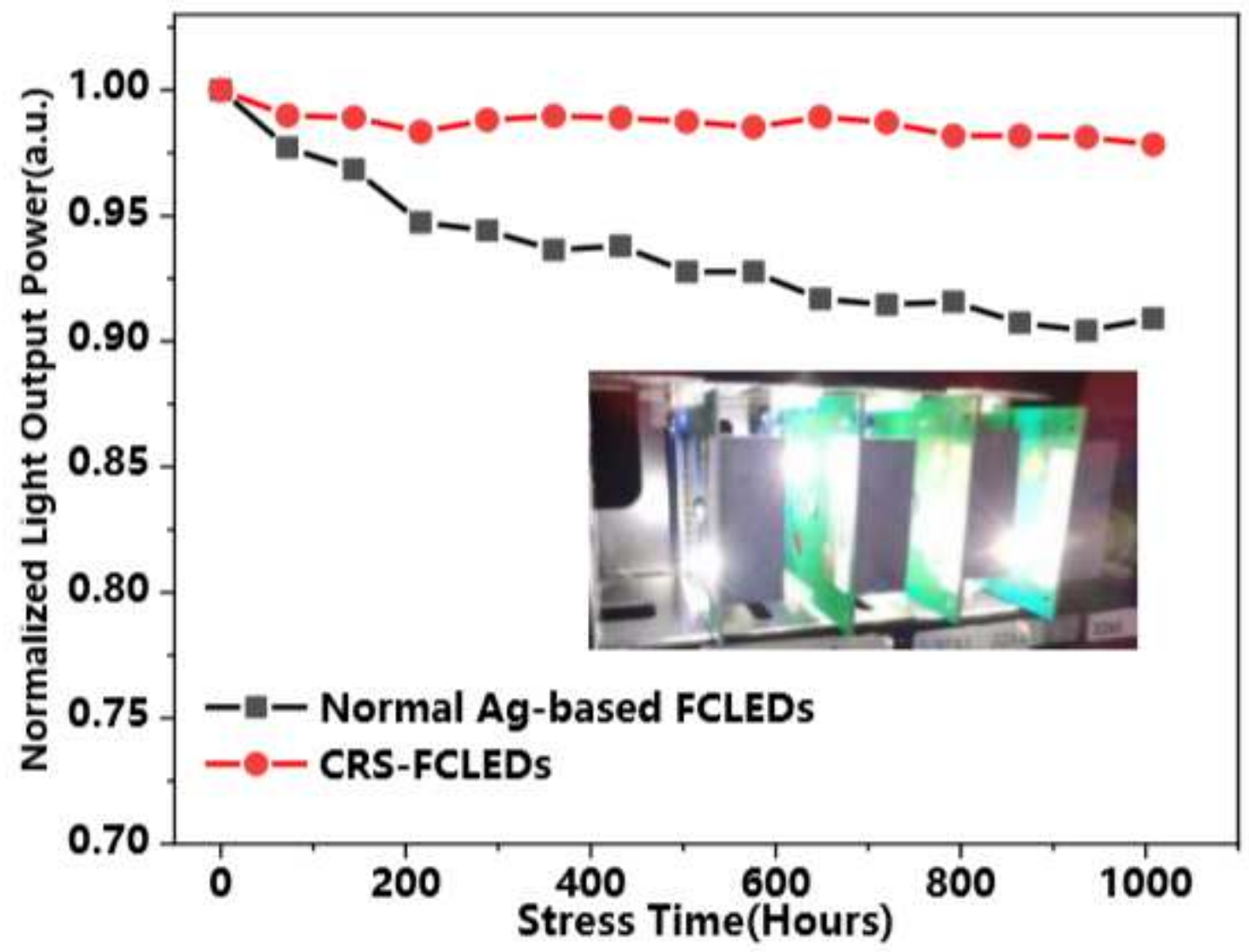

Figure 7

Optical degradation of CRS-FCLED and normal Ag-based FCLED during high temperature operation life test. 